\title{
Analysis of Electron Mobility in Some "Problematic Materials" from Magnetoresistance Effect at High Magnetic Fields
}

\author{
V.V. Shchennikov ${ }^{a}$, A.E. Kar'Kin ${ }^{a}$, S.V. Ovsyannikov ${ }^{b, c}$ and N.V. Morozova ${ }^{a, *}$ \\ ${ }^{a}$ Institute of Metal Physics of Russian Academy of Sciences, Urals Division \\ 18 S. Kovalevskaya Str., Yekaterinburg 620990, Russia \\ ${ }^{b}$ Bayerisches Geoinstitut, Universität Bayreuth, Universitätsstr. 30, Bayreuth D-95447, Germany \\ ${ }^{c}$ Institute for Solid State Chemistry of Russian Academy of Sciences, Urals Division \\ 91 Pervomayskaya Str., Yekaterinburg 620049, Russia
}

\begin{abstract}
Using examples of some perspective electronic materials ( $\mathrm{HgSeS}, \mathrm{Fe}_{3} \mathrm{O}_{4}$, InN, and others) it is shown that magnetoresistance data at high magnetic fields allow evaluating the true values of mobility of charge carriers in spite of any "adverse factors". Additional impacts involving high pressure and irradiation with high-energy particles (neutrons, electrons, ions) produce the enhancement of magnetoresistance technique of testing and allow to go into details of the type of electron structure and scattering mechanisms of charge carriers.
\end{abstract}

PACS: 72.20.My, 62.50.-p, 61.80.-x, 72.20.Jv

\section{Introduction}

Precise values of charge carrier parameters of electronic materials, namely their mobility and concentration, present crucial factors for application at electronic devices [1-10]. Determination of concentration and mobility of charge carriers even in case of two-band conductivity requires measurements of at least four different experimental effects [2]. For inhomogeneous materials, the determination of electron mobility from the Hall effect presents a serious problem even in one-band case because of inclusions affecting the conductivity [4]. The magnetoresistance effect is known to carry the information on the conduction mechanism and mobilities of the most mobile charge carriers [11]. Values of mobility found from magnetoresistance (MR) effect in particular materials exceed those obtained from the Hall effect by $\approx 10^{2}-10^{4}$ times $[3,4]$ and the former seem to be close to bona fide values.

In the present work we examined the carrier mobility in some "problematic materials" with various kinds of adverse factors ( $\mathrm{HgSeS}, \mathrm{Se}, \mathrm{Fe}_{3} \mathrm{O}_{4}, \mathrm{Ti}_{2} \mathrm{O}_{3}$ and $\mathrm{InN}$ ) using magnetoresistance data.

\section{Experiment}

MR effect was measured in the static magnetic field $B$ of up to $\approx 14 \mathrm{~T}$ and at temperatures of $4.2-370 \mathrm{~K}$, using

\footnotetext{
* corresponding author; e-mail: natasha_nt88@mail.ru, highpressgroup@mail.ru
}

Oxford Instruments equipment [5, 6]. Measurements at the ambient pressure were performed by the conventional Montgomery method (a version of the Van der Pauw method), allowing anisotropy examination of galvanomagnetic properties [7]. For the investigations, we took single crystals ( $\mathrm{HgSeS}, \mathrm{Fe}_{3} \mathrm{O}_{4}$ ), amorphous samples (Se), polycrystalline bulk $\left(\mathrm{Ti}_{2} \mathrm{O}_{3}\right)$, and thin film (InN) crystals. Compositions of the samples were determined by means of X-ray microprobe analysis using a Superprobe-JCXA-733 spectrometer (JEOL, Japan). The samples of magnetite $\left(\mathrm{Fe}_{3} \mathrm{O}_{4}\right)$ were subjected to a fast neutron bombardment with a fluency of $\Phi=2.2 \times 10^{20} \mathrm{~cm}^{-2}$ [8]. High hydrostatic pressure up to $\approx 2 \mathrm{GPa}[9]$ as well as quasi-hydrostatic pressure up to $\approx 30 \mathrm{GPa}$ [10] were used also as additional tuning parameters (for $\mathrm{HgSeS}$ and Se).

\section{Results}

The collected results for all the materials are presented in Figs. 1-6. The obtained dependences of MR on magnetic field in most of the materials may be described by the following equation:

$$
\frac{\Delta \rho}{\rho}=D \frac{(\mu B)^{2}}{1+(\mu B)^{2}},
$$

where $\rho$ is the electrical resistivity, $B$ is the magnetic induction, $\mu$ is charge carriers mobility, coefficient $D$ determines the sign and value of MR. Both positive $(D>0)$ and negative effects $(D<0)$ were found $[1,11]$. The resultant sign of MR effect depends on contributions of both the negative and positive MR effects induced by the peculiarities of the electronic band structure as well as the mechanisms of charge carriers scattering [11]. 

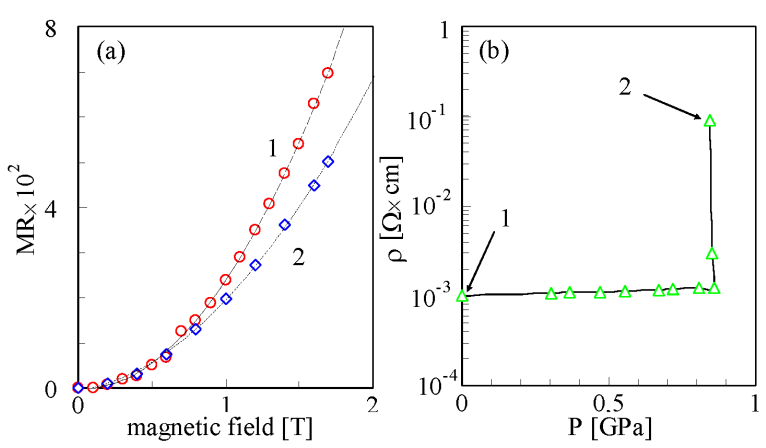

Fig. 1. (a) Dependences of magnetoresistance effects on magnetic field for $\mathrm{HgSe}_{1-x} \mathrm{~S}_{x}$ with $x=0.104$ at $T=77 \mathrm{~K}$ at $P=0(1)$ and $P=0.84 \mathrm{GPa}(2)$; (b) dependence of electrical resistivity on hydrostatic pressure for the same sample; the MR data (a) have been obtained at points 1 and 2 [13].

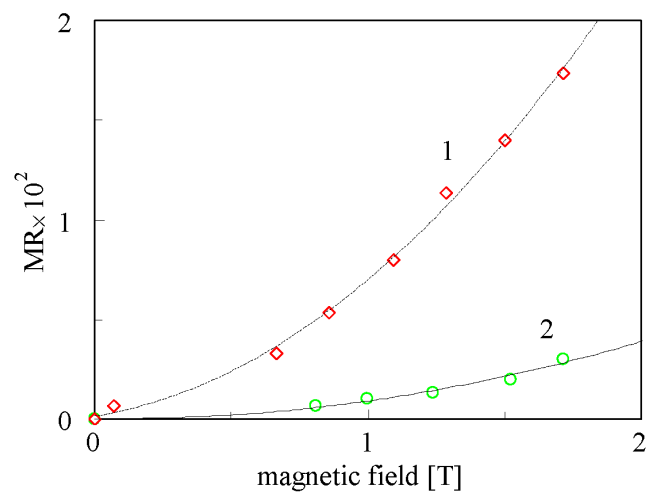

Fig. 2. Dependences of the magnetoresistance effects of amorphous Se on magnetic field at $T=290 \mathrm{~K}$ under pressure $23 \mathrm{GPa}(1)$ and $12 \mathrm{GPa}(2)$ [1].

\subsection{High pressure phases and two-phase states}

HgSe-based crystals undergo a phase transition from a semimetal cubic zinc-blende phase (B3) to a semiconducting hexagonal cinnabar (B9) one under pressure. The samples of $\mathrm{HgSeS}$ consisted of these two phases in an intermediate region of hydrostatic pressure (Fig. 1) $[3,12,13]$. The value of $\rho$ of the samples in the two-phase state increased by $2-4$ orders of magnitude (Fig. 1), but the positive sign of the temperature coefficient of $\rho$ remained [3, 4], and the MR retained almost the same as for the initial phase (Fig. 1). The MR effect in the two-phase state was therefore affected by inclusions of the initial semimetal phase. At the completion of the phase transition to the hexagonal phase, the MR effect became negative (for all $\mathrm{HgX}$, where $\mathrm{X}=\mathrm{Te}$, Se, S, O) [14, 15]. Under the pressure increase above $\approx 20 \mathrm{GPa}$, the phase transition to a metallic high-pressure phase happened. At this transition, the MR effect showed a tendency to change its sign to positive [15]. The decrease of pressure leads to the opposite variations of both the sign and the values of the MR effect [14].

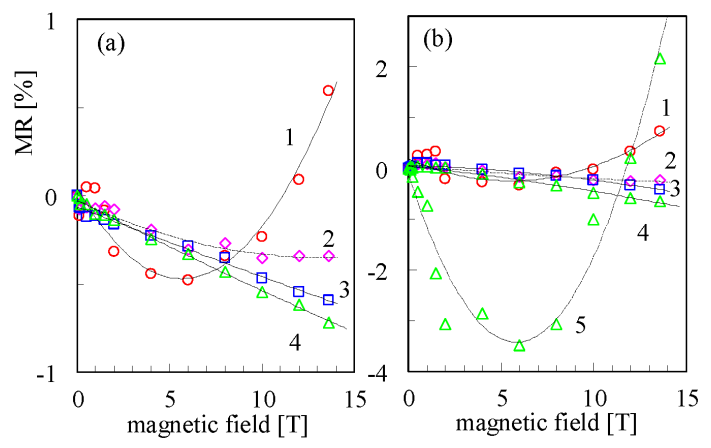

Fig. 3. Dependences of the magnetoresistance effects on magnetic field for as-grown (a) and fast-neutron-bombarded $\mathrm{Fe}_{3} \mathrm{O}_{4}$ (b) at $T=140 \mathrm{~K}(1), T=200 \mathrm{~K}(2)$, $T=305(3), T=370(4)$ and $T=110 \mathrm{~K}(5)$ [8].

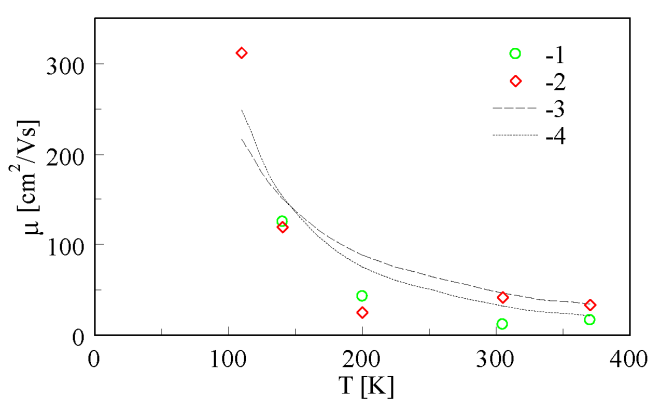

Fig. 4. Dependences of mobility values of $\mathrm{Fe}_{3} \mathrm{O}_{4}$ on temperature for: 1 - as-grown, and 2 - fast-neutron-bombarded samples; the points are data estimated from the MR effects (Fig. 3), and the lines are theoretical approximations: $3-\mu \sim T^{-3 / 2}, 4-\mu \sim T^{-2}$.

Notice that in wide-gap semiconductors, such as GaP, $\mathrm{ZnSe}, \mathrm{ZnS}, \mathrm{CdS}, \mathrm{CdSe}$, and $\mathrm{CdTe}$, the resistivity decreases at phase transitions into a rock-salt lattice by $\approx 6-7$ orders of magnitude, and the magnetoresistance was found to be negative with a nearly parabolic dependence on $B[1,14,15]$. Classical positive MR has to be small in these metal phases due to factor of $D=\left(k_{\mathrm{B}} T / \eta\right)^{2} \ll 1[11,1]$ ( $\eta$ is the chemical potential, $\eta \gg k_{\mathrm{B}} T, k_{\mathrm{B}}$ is the Boltzmann constant, $T-$ temperature), so the negative MR dominated. The dependence of the negative MR-effect on magnetic field and its rising with lowering in temperature suggested that it may be due to one of the following reasons: (i) the "hot" or "warm" electrons, which are "cooled" by the magnetic field [11], (ii) interference quantum additions to the conductivity [14], or (iii) by impurity levels splitting in the magnetic field [16]. We can conclude that the MR data showed the charge carrier dominating scattering mechanisms at various high-pressure phases.

\subsection{Inhomogeneous semiconductors}

In trigonal Se, the Hall effect data showed low mobility values of holes, $\mu \approx 0.01 \mathrm{~cm}^{2} /(\mathrm{Vs})$, while direct $\mathrm{MR}$ measurements gave much higher values of $\mu \approx$ 

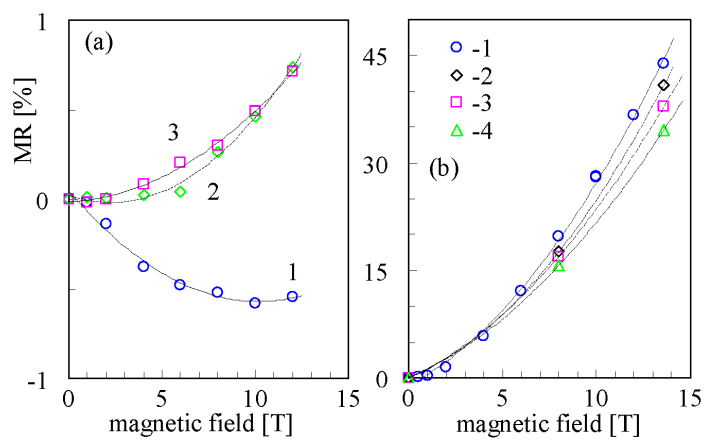

Fig. 5. Dependences of the magnetoresistance effects on magnetic field for the gold phase of $\mathrm{Ti}_{2} \mathrm{O}_{3}$ (a): at $T=247.2 \mathrm{~K}(1), T=296.5 \mathrm{~K}(2), T=346 \mathrm{~K}(3)$ and for InN thin film (b) at $T=4.2 \mathrm{~K}(1), T=107 \mathrm{~K}(2)$, $T=206 \mathrm{~K}(3)$, at $286 \mathrm{~K}(4)$. The points are experimental data and the lines are fits by polynomial (a) and quadratic (b) approximations.
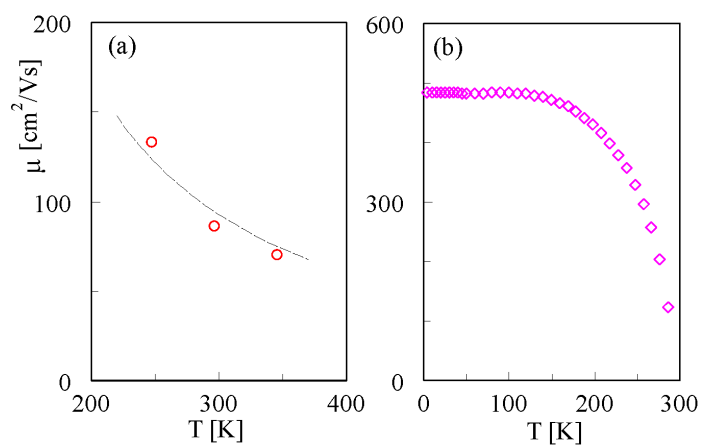

Fig. 6. Dependences of mobility values on temperature for $\mathrm{Ti}_{2} \mathrm{O}_{3}$ (a) and InN (b). The points are data estimated from Fig. 5, the line shows a fit by the function $\mu \sim T^{-3 / 2}$.

30-50 $\mathrm{cm}^{2} /(\mathrm{V} \mathrm{s})$ [4]. This divergence is related to the worsening of the electrical resistivity due to intrinsic potential barriers in the material [4]. In our measurement, the mobility values $\left(\mu \approx 68 \mathrm{~cm}^{2} /(\mathrm{Vs})\right.$ at $P=23 \mathrm{GPa}$ and $\approx 32 \mathrm{~cm}^{2} /(\mathrm{Vs})$ at $\left.P=12 \mathrm{GPa}\right)$ estimated from the MR data (Fig. 2) increased under pressure, suggesting a closing of direct energy gap at $P \approx 25 \mathrm{GPa}[10]$. For direct-energy-gap semiconductor Se (its gap is at $H$-point of the Brillouin zone) $\mu \sim 1 / m \sim 1 / E_{\mathrm{g}}$, where $m$ is the effective mass of charge carriers and $E_{\mathrm{g}}$ is the semiconductor gap $[17,18]$. The exponential increase of the MR effect under pressure is related to the decrease of $E_{\mathrm{g}}$ and $m$ (Fig. 2). Thus, the positive MR effect characterizes the mobility $\mu(P)$ of the light carriers [11], and hence it gives information about the type of semiconductor gap $E_{\mathrm{g}}$ (direct gap at high pressures) [17, 18]. Similar results have been obtained for other direct-gap semiconductors Te [14], and PbTe, PbSe [19, 20]. For sulphur, the gap at $P \approx 40 \mathrm{GPa}$ was found from the MR measurements to be indirect [21].

\subsection{Material with magnetic ordering}

The high degree of magnetization and resultant large anomalous Hall effect in magnetite are serious obstacles for the examination of ordinary MR effect [8]. At high magnetic fields above $B>3 \mathrm{~T}$, the correct measurements of MR effects become available (Fig. 3). In the temperature range $100-300 \mathrm{~K}$ there are both positive and negative contributions to the MR effects of magnetite. The negative MR effect may be attributed to the suppression of scattering of electrons with spin flip [22]. The equation for the negative MR effect for this case is the following:

$$
\frac{\Delta \rho}{\rho_{0}} \approx-\left(\frac{g S \beta B}{T k_{\mathrm{B}}}\right)^{2},
$$

where $g \approx 2, \beta=9.27 \times 10^{-24} \mathrm{~J} / \mathrm{T}$ is the Bohr magneton, $S$ is the value of spin [23]. The increase of the degree of magnetic ordering with increasing $B$ is known to reduce electron scattering (and, hence - the electrical resistivity). Below the Verwey temperature, an absolute value of the positive magnetoresistance effect increases in accordance with the higher mobility of holes in the low-temperature phase [8]. A temperature dependence of the mobility obtained from the positive MR effect of magnetite can be described by a function of $\mu \sim T^{-3 / 2}$ (Fig. 4), which corresponds to the scattering of charge carriers by acoustic phonons [11]. The hole mobility value decreased after neutron irradiation, but its value still remained rather high (as in trigonal Se). This result confirmed the Peierls model of the Verwey transition in $\mathrm{Fe}_{3} \mathrm{O}_{4}$, but did not support the charge-ordering model of this transition [8].

\subsection{Two-band conductivity}

A localized donor state, resonant with the conduction band, influences electrical conductivity in $\mathrm{InN}$ samples $[23,24]$. Above $\approx 140 \mathrm{~K}$, the mobility value estimated from the MR effect (Fig. 5) in the thin films studied (of $\approx 1 \mu \mathrm{m}$ thickness) decreases, suggesting a dominant acoustic phonon scattering at high temperatures [25], while at low temperatures the mobility was independent of temperature (Fig. 6).

The Hall effect data of $\mathrm{Ti}_{2} \mathrm{O}_{3}$ (in so-called "golden" phase synthesized at high pressure-high temperature (HP-HT) conditions) in magnetic fields up to $14 \mathrm{~T}$ indicated a two-band model of electrical conductivity [26]. In weak-magnetic-field approximation $(\mu B<1)$ the magnetoresistance effect $(\Delta \rho / \rho)$ for two-band semiconductor comes as follows [11]:

$$
\frac{\Delta \rho}{\rho}=B^{2} b_{r}\left[\left(\frac{\sigma_{n}}{\sigma} \mu_{n}^{2}+\frac{\sigma_{p}}{\sigma} \mu_{p}^{2}\right)-\left(\frac{\sigma_{n}}{\sigma} \mu_{n}-\frac{\sigma_{p}}{\sigma} \mu_{p}\right)^{2} \frac{a_{r}^{2}}{b_{r}}\right],
$$

where $\sigma_{n}$ and $\sigma_{p}$ are the electron and hole partial contributions, respectively to the total electrical conductivity $\sigma ; a_{r}$ and $b_{r}$ are the constants, depending on scattering parameter $r$ defining the dependence of relaxation time $\tau$ on electron energy $\varepsilon: \tau(\varepsilon) \sim \varepsilon^{r}$. For semimetals, the MR effect may be considered by analogy with simple 
one-band case as follows $[11,19]: \mathrm{MR} \sim\left(\mu_{\mathrm{eff}} B\right)^{2}$, where $\mu_{\mathrm{eff}}=\left(\mu_{n} \mu_{p}\right)^{1 / 2}$ is an "effective" mobility that accounts contributions from electron and holes bands (Fig. 5). The carrier mobility values in $\mathrm{Ti}_{2} \mathrm{O}_{3}$ samples rapidly decreased with increasing temperature (Fig. 6), which is characteristic for the intrinsic conductivity with phonon scattering mechanism of charge carriers $\left(\mu \sim T^{-3 / 2}\right)[11]$.

\section{Conclusions}

The MR effect depends strongly on both the magnetic field and the mobility value of the most mobile carriers $\left(\mathrm{MR} \sim(\mu B)^{2}\right)$ and therefore it allows a direct determination of $\mu$ in "problematic materials", that include (but are not limited to) inhomogeneous semiconductors, mixture of phases, materials with magnetic ordering, two-band conductivity, and others. Thus, MR data allow to reveal the electron structure (direct or indirect semiconductor gap) and charge carriers scattering mechanisms. The influences tending to the negative contribution to MR effect usually have a weaker dependence on $B$ and may be ruled out at high fields (e.g., saturation of magnetization at $\mathrm{Fe}_{3} \mathrm{O}_{4}$ ). Additional impacts like high-energy particles bombardment and high pressure enhance the power of the MR method of testing.

\section{Acknowledgments}

The work was partly supported by the RFBR grant and by the Program of the Presidium of RAS.

\section{References}

[1] V.V. Shchennikov, in: High Pressure Science and Technology, Proc. Joint XV ARAPT and EHPRG Int. Conf., Ed. W.A. Trzeciakowski, World Sci., Singapore 1996, p. 490.

[2] C.C. Kwan, J. Basinski, J.C. Woolley, Phys. Status Solidi B 48, 699 (1971).

[3] V.V. Shchennikov, N.N. Gavaleshko, V.M. Frasunyak, V.I. Osotov, Phys. Solid State 37, 1311 (1995).

[4] H. Mell, J. Stuke, Phys. Status Solidi 24, 183 (1967).

[5] A.E. Kar'kin, V.V. Shchennikov, S.V. Ovsyannikov, E.P. Skipetrov, B.N. Goshchitskii, Tech. Phys. Lett. 30, 328 (2004).

[6] S.V. Ovsyannikov, V.V. Shchennikov, A.E. Kar'kin, B.N. Goshchitskii, J. Phys., Condens. Matter 17, S3179 (2005).

[7] A.E. Kar'kin, B.N. Goshchitskii, Phys. Particles Nuclei 37, 807 (2006).
[8] V.V. Shchennikov, S.V. Ovsyannikov, A.E. Karkin, S. Todo, Y. Uwatoko, Solid State Commun. 149, 759 (2009).

[9] V.V. Shchennikov, A.E. Kar'kin, N.P. Gavaleshko, V.M. Frasunyak, Phys. Solid State 42, 215 (2000).

[10] V.V. Shchennikov, V.I. Osotov, Phs. Solid State 37, 243 (1995).

[11] K. Seeger, Semiconductor Physics, Springer Verlag, Berlin 1984.

[12] V.V. Shchennikov, A.E. Kar'kin, N.P. Gavaleshko, V.M. Frasunyak, Phys. Solid State 39, 1528 (1997).

[13] S.V. Ovsyannikov, V.V. Shchennikov, I.A. Komarovskii, G.V. Vorontsov, I.V. Korobeynikov, V.V. Shchennikov Jr., Proc. SPIE 7978, 79781W (2011).

[14] V.V. Shchennikov, Phys. Solid State 35, 401 (1993).

[15] V.V. Shchennikov, "Magnetoresistance of mercury, cadmium and zinc chalcogenides at ultra-high pressure" (paper is deposited at VINITI No. 1016-B 89), Institute of Metal Physics, Urals Division, Academy of Sciences, USSR, Sverdlovsk 1989, p. 1.

[16] S.A. Obukhov, Preprint No. 1459, A.F. Ioffe Physico-technical Institute, Academy of Sciences of the USSR, Leningrad 1991.

[17] V.V. Shchennikov, Defect Diffus. Forum 208-209, 275 (2002).

[18] V.V. Shchennikov, S.V. Ovsyannikov, Solid State Commun. 121, 323 (2002)

[19] V.V. Shchennikov, S.V. Ovsyannikov, Solid State Commun. 126, 373 (2003).

[20] V.V. Shchennikov, S.V. Ovsyannikov, JETP Lett. 77, 88 (2003).

[21] V.V. Shchennikov, S.V. Ovsyannikov, Phys. Status Solidi B 239, 399 (2003).

[22] A.A. Abrikosov, Fundamental of the Metal Theory, Nauka, Moscow 1987.

[23] L.H. Dmowski, K. Dybko, J. Plesiewicz, T. Suski, H. Lu, W. Schaff, M. Kurouchi, Y. Nanishi, L. Konczewicz, V. Cimalla, O. Ambacher, Phys. Status Solidi $B$ 243, 1537 (2006).

[24] T. Suski, A. Kamińska, G. Franssen, H. Teisseyre, L.H. Dmowski, J.A. Plesiewicz, H.L. Lu, W.J. Schaff, M. Kurouchi, Y. Nanishi, Phys. Status Solidi B 244, 1825 (2007)

[25] S.V. Ovsyannikov, V.V. Shchennikov, A.E. Karkin, A. Polian, O. Briot, S. Ruffenach, B. Gil, M. Moret, Appl. Phys. Lett. 97, 032105 (2010).

[26] S.V. Ovsyannikov, X. Wu, V.V. Shchennikov, A.E. Karkin, N. Dubrovinskaia, G. Garbarino, L. Dubrovinsky, J. Phys., Condens. Matter 22, 375402 (2010). 